\title{
Exposição dérmica do aplicador de agrotóxicos na cultura da uva, com diferentes pulverizadores ${ }^{1}$
}

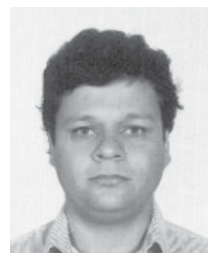

\author{
Hamilton H. Ramos ${ }^{2}$, José V. G. Maziero², Kiyoshi Yanai², Ila M. Corrêa², Francisco J. Severino ${ }^{3}$, \\ Oscar Y. Kanno ${ }^{3}$, Paulo S. Martins ${ }^{3}$, Cláudio Mura ${ }^{3} \&$ Marcelo A. Morgano ${ }^{4}$
}

\begin{abstract}
1 Trabalho integrante das atividades do Programa Segurança e Saúde do Trabalhador Rural
2 Pesquisador Científico, Centro de Mecanização e Automação Agrícola (CMAA), Instituto Agronômico (IAC). C.P. 26, CEP 13201-970, Jundiaí, SP. Fone: (11) 4582-8155, Fax: (11) 4582-8184. E-mail: hhramos@dea.iac.br (Foto)

${ }^{3}$ Engenheiro Agônomo, Coordenadoria de Assistência Técnica Integral, EDR-Dracena. CP 164, CEP 17900-000, Dracena, SP. Fone: (18) 5821-3250, Fax: (18) 5821-3294. E-mail: catidracena@fundec.com.br

4 Pesquisador Científico, Centro de Química de Alimentos e Nutrição Aplicada, Instituto de Tecnologia de Alimentos, CP 139, CEP 13073-001, Campinas, SP. Fone: (19) 3743-1700. E-mail: morgano@ital.org.br
\end{abstract}

Protocolo $151-31 / 10 / 2001$

\begin{abstract}
Resumo: Com este trabalho, objetivou-se avaliar a exposição do aplicador à calda de agrotóxico, durante pulverização na cultura da videira, com 4 diferentes pulverizadores. Os equipamentos avaliados foram um pulverizador semi-estacionário típico, um pulverizador de barras adaptado a uma barra semi-circular com os bicos voltados para cima, um turbopulverizador marca Hatsuta modelo café, com sistema de turbina adaptado para utilização na cultura da uva, e um turbopulverizador marca KO, modelo Jales, regulados pelos produtores. Para avaliação da exposição dérmica potencial (EDP) amostradores foram distribuídos sobre 22 partes do corpo do aplicador, que pulverizou uma calda contendo 3000 ppm do cátion $\mathrm{Cu}^{+2}$ durante 35 a $40 \mathrm{~min}$. Em laboratório o $\mathrm{Cu}^{+2}$ foi recuperado e quantificado e a exposição calculada em função da concentração do cátion na calda. O pulverizador semi-estacionário proporcionou a maior EDP, seguido pela barra, turbo adaptado e pelo turbo original. A porcentagem do volume pulverizado que atinge o corpo do aplicador num mesmo período de tempo, foi mais que 89 vezes maior para o semi-estacionário, quando comparado ao turbo Jales e à barra adaptada, e 44 vezes maior quando comparado ao turbo adaptado. As áreas mais expostas do corpo foram mãos, cabeça e dorso para os tratorizados e a EDP foi alta em praticamente todas as regiões do corpo com o semi-estacionário. Aspectos da EDP em função do equipamento e da forma de utilização são discutidos.
\end{abstract}

Palavras-chave: agrotóxicos, pulverizador, exposição dérmica, uva

\section{Dermal exposure of pesticide applicator in grapes crop, with different sprayers}

\begin{abstract}
The aim of present study was to evaluate the dermal exposure of pesticide applicator, during spraying in grapes crop, with 4 different sprayers. The equipments utilized were a typical semi-stationery sprayer, a boom sprayer adapted with a semi-circulate boom with the nozzle returned upward, an airblast sprayer utilized in coffee crop with turbine system adapted for grapes and a original airblast sprayer for grapes (KO, model Jales), regulated by the workers. For assessment of potential dermal exposure (PDE) of spray man, 22 sampling pads were attached to body parts of spray man applying a solution containing $3000 \mathrm{ppm}$ of $\mathrm{Cu}^{+2}$ during 35 to $40 \mathrm{~min}$. Copper was extracted and quantified in laboratory and the exposure was calculated considering the original concentration of the spray solution. The semi-stationery sprayer provided largest PDE, followed by the adapted boom sprayer, adapted airblast and original airblast sprayer. The carrier percentage reaching the spray man body in the same period of time was more than 89 times higher for semi-stationery when compared with the original airblast and the adapted boom and 44 times higher compared to the adapted airblast sprayer. The most exposed areas of the spray man body were the hands, head and back for sprayers adapted to tractor, and PDE was high in practically all the areas of the body with the semi-stationery equipment. Aspects of PDE related to equipment and the form of their use are discussed.
\end{abstract}

Key words: pesticides, sprayer, dermal exposure, grapes 


\section{INTRODUÇÃO}

Segundo dados do IBGE (1999) o Estado do Rio Grande do Sul ainda é o maior produtor brasileiro de uvas para vinho, além de responsável por $92 \%$ da produção; no entanto, o Estado de São Paulo já responde por $56 \%$ da produção de uva de mesa, constituindo-se no maior produtor brasileiro neste setor. Outros estados brasileiros também possuem produções bastante significativas, destacando-se Santa Catarina e Paraná e o Nordeste brasileiro, mais especificamente a região do Vale do Rio São Francisco.

Por se tratar de uma planta sarmentosa, a videira necessita de tutores para sua condução, sendo dois os tipos principais de apoio: caramanchão (latada ou pérgola) e espaldeira (casca). Segundo Simão (1971) as videiras conduzidas em caramanchão são mais produtivas, porém os frutos são inferiores e mais sujeitos a doenças causadas por fungos; portanto, tal sistema de condução deve destinar-se principalmente ao cultivo doméstico e à ornamentação. Tal idéia não é compartilhada por Maruyama (1980) que recomenda que a videira seja conduzida em "latada", pois assim ela produzirá mais e melhor. No Estado de São Paulo ambos os sistemas de condução são comuns, sendo a espaldeira mais utilizada nas regiões produtoras de Jundiaí e São Roque e o caramanchão nas regiões de Jales, São Miguel Arcanjo e Capão Bonito, por proporcionar maior proteção dos cachos contra a queimadura pelo sol.

Qualquer que seja o sistema de condução, o método mais comum de controle das pragas e doenças que ocorrem na cultura, é a aplicação de agrotóxicos através de pulverizações (Ramos et al., 1998). Segundo Maruyama (1980) o número de pulverizações anuais varia em função da susceptibilidade da variedade, podendo variar de 5 a 10 em variedades como a Niagara, até 40 a 50 em outras, como a Itália. O equipamento mais comumente utilizado nessas pulverizações é o pulverizador semi-estacionário (Ramos et al., 1998), em que o tanque e a bomba ficam parados nos carreadores ou em uma posição fixa do terreno e enviam calda pressurizada para duas mangueiras de alta pressão, de diâmetro interno (8 ou $12,7 \mathrm{~mm}$ ) e comprimentos variáveis (50 a $100 \mathrm{~m})$. Cada mangueira pode, ainda, ser subdividida em duas, logo após a saída da bomba possibilitando, assim, que quatro operários trabalhem ao mesmo tempo. Em cada extremidade das mangueiras é acoplada uma lança, com aproximadamente $1 \mathrm{~m}$ de comprimento, contendo uma ponta com 3 bicos leque dispostos em linha (Yamaho) ou 3 bicos cônicos dispostos triangularmente (Bico Universal Hatsuta) trabalhando a pressões superiores a $1400 \mathrm{kPa}$. Durante a aplicação, o operário empunha a lança direcionando a pulverização para o lado e movimentando-a para cima e para baixo, nos plantios em espaldeira, ou direcionando a pulverização para cima e movimentando-a lateralmente, nos plantios em caramanchão. Em ambos os casos, o operário passa pela área tratada imediatamente após a pulverização. Apesar da predominância do semi-estacionário, outros pulverizadores, normalmente adaptados pelos produtores buscando mecanizar a operação, são também freqüentemente encontrados.

A exposição dérmica pode ser potencial (EDP) que é a quantidade de agrotóxico coletada sobre a pele exposta, sobre as roupas, luvas protetoras etc. que, teoricamente, atingirá a pele na ausência das vestimentas (Turnbull et al., 1985); ou real (EDR) que é a quantidade absoluta de um agrotóxico qualquer que entra em contato com a pele (sem roupa) e, portanto, disponível para a absorção (Bonsall, 1985). Segundo Durham \& Wolfe (1962) numerosos métodos têm sido desenvolvidos para medir a exposição de aplicadores de agrotóxicos e outros agentes envolvidos, os quais podem ser convenientemente divididos em métodos diretos e indiretos. Os diretos envolvem o uso de algum mecanismo para coletar o material tóxico que vem em direção e entra em contato com o corpo do aplicador, durante o período de exposição. A quantidade do agente tóxico coletado é determinada por análises químicas, bioensaios, ou outros métodos. Os métodos indiretos, por outro lado, envolvem a medida de algum efeito do agente tóxico no indivíduo. $\mathrm{O}$ método direto foi utilizado nos primeiros estudos na área e originou o Protocolo Padrão da Organização Mundial de Saúde de 1975 (WHO, 1975), modificado em 1982 (WHO, 1982). Tal método, adaptado por Machado Neto \& Matuo (1989) tem sido utilizado no Brasil em estudos mais recentes de avaliação (Machado Neto et al., 1992; Machado Neto et al., 1998).

O presente trabalho tem como objetivo avaliar a exposição dérmica potencial do aplicador à calda de agrotóxico, obtida pela utilização de 4 diferentes sistemas de pulverização utilizados na cultura da videira.

\section{MATERIAL E MÉTODOS}

Preliminarmente ao ensaio de exposição, realizou-se uma avaliação dos sistemas de aplicação utilizados pelos produtores de uva na região de Dracena, SP, tendo-se selecionado, para estudo, os 4 equipamentos mais representativos (Figura 1) que foram: um pulverizador semi-estacionário típico, um pulverizador de barras adaptado a uma barra semi-circular de $2 \mathrm{~m}$ com 8 bicos espaçados $25 \mathrm{~cm}$ e voltados para cima, um turbo pulverizador marca Hatsuta modelo café, com sistema de turbina adaptado na região para utilização na cultura da uva e um turbopulverizador marca KO, modelo uva, com configuração original. Os pulverizadores, juntamente com os tratores e
A.

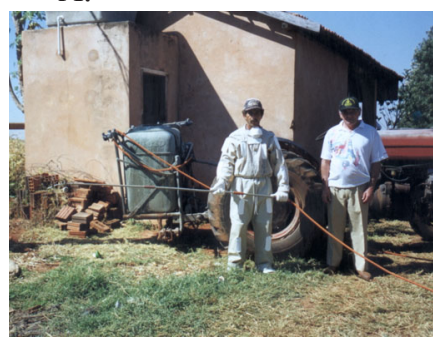

C.

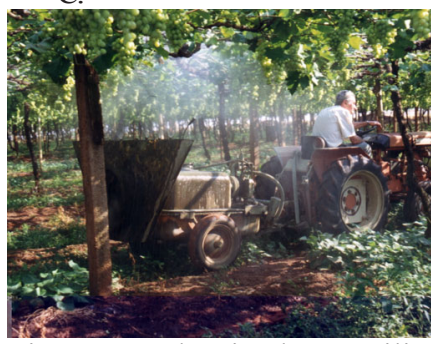

B.

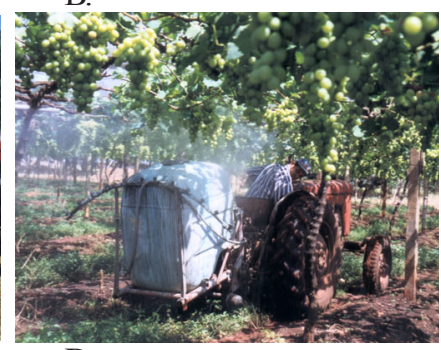

D.

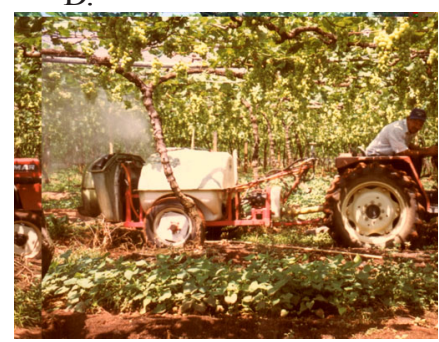

Figura 1. Pulverizadores utilizados na avaliação da exposição dérmica do aplicador na cultura da uva. (A) pulverizador semiestacionário; (B) pulverizador de barras adaptado; (C) turbopulverizador adaptado; (D) turbopulverizador 
Tabela 1. Descrição dos tratamentos utilizados no ensaio de exposição dérmica do aplicador de agrotóxicos na cultura da uva

\begin{tabular}{|c|c|c|c|c|c|c|c|}
\hline \multirow[b]{2}{*}{ Tratamento } & \multirow[b]{2}{*}{ Pulverizador } & \multirow[b]{2}{*}{ Trator } & \multirow[b]{2}{*}{$\begin{array}{l}\text { Velocidade } \\
\left(\mathrm{km} \mathrm{h}^{-1}\right)\end{array}$} & \multicolumn{3}{|c|}{ Bicos de Pulverização } & \multirow{2}{*}{$\begin{array}{l}\text { Volume } \\
\left.(\mathrm{L} \mathrm{ha})^{-1}\right)\end{array}$} \\
\hline & & & & Tipo & $\mathrm{N}^{\mathrm{o}}$ & $\begin{array}{l}\text { Vazão Média } \\
\left(\mathrm{L} \min ^{-1}\right)\end{array}$ & \\
\hline $\mathrm{T} 1$ & Turbo adaptado & Agrale 4100 & 2,27 & Jacto JD2 & 10 & 0,784 & 689 \\
\hline $\mathrm{T} 3$ & Barra adaptada & MF $50 \mathrm{X}$ & 5,55 & MAG 11003 & 12 & 2,175 & 940 \\
\hline $\mathrm{T} 4$ & Semi-estacionário & MF $50 \mathrm{X}$ & 1,11 & Yamaho D5 & 3 & 0,997 & 542 \\
\hline
\end{tabular}

operadores foram, então, transportados a uma mesma área experimental, para que as avaliações pudessem ser realizadas concomitantemente.

As avaliações da exposição dérmica do aplicador à calda de pulverização foram realizadas no Sítio Esporte, localizado em Ouro Verde, SP, e pertencente ao Sr. Sérgio Martins. A área experimental foi constituída por 1,0 ha de uva da variedade Rubi, em estádio de início do amolecimento das bagas, plantados em caramanchão, no espaçamento de 3,0 x 6,0 m.

Os tratamentos utilizados foram os pulverizadores, operando nas condições de conservação e operação em que se encontravam. Previamente ao início das avaliações, os pulverizadores receberam uma lavagem para descontaminação e os produtores demonstraram a forma como operavam os equipamentos, tendo-se avaliado a velocidade, a vazão da barras de bicos e volume de aplicação. Os tratamentos utilizados são descritos na Tabela 1 .

Durante as avaliações, os aplicadores usaram roupa própria para aplicação de agrotóxicos, de tecido de brim leve, hidrorrepelente e antiaderente, devido ao tratamento prévio do fio com Teflon, e aprovada pelo Ministério do Trabalho como equipamento de proteção individual. Tal vestimenta, no entanto, foi utilizada apenas como suporte para fixação dos amostradores.

Os coletores utilizados foram absorventes higiênicos femininos, marca Carefree, e luvas de algodão, conforme avaliaram Machado Neto \& Matuo (1989) e os utilizaram Machado Neto et al. (1992) e Machado Neto (1997). Os coletores foram afixados sobre as roupas em 22 diferentes partes do corpo do aplicador (Tabela 2 e Figura 2) selecionadas por Machado Neto et al. (1992).

Tabela 2. Partes do corpo diferenciadas e locais do corpo amostrados, para avaliação da exposição dérmica com as respectivas áreas

\begin{tabular}{llr}
\hline Partes do Corpo & \multicolumn{1}{c}{ Locais Amostrados } & Área $\left(\mathrm{cm}^{2}\right)$ \\
\hline Cabeça & Cabeça & $1.100,0$ \\
Rosto & Face & 740,0 \\
"V" do pescoço & "V" do pescoço & 150,0 \\
Braço & Direito e Esquerdo & $1.250,0$ \\
Antebraço & Direito e Esquerdo & 625,0 \\
Mão & Direita e Esquerda & 820,0 \\
Dorso & Costas & $3.515,0$ \\
Peito & Direito e Esquerdo & 625,0 \\
Coxa & Direita e Esquerda/Frente e Atrás & 878,5 \\
Perna & Direita e Esquerda/Frente e Atrás & 593,5 \\
Pé & Direito e Esquerdo & 585,0 \\
\hline Total & & $19.203,0$ \\
\hline Fonte: (Machado Neto et al., &
\end{tabular}
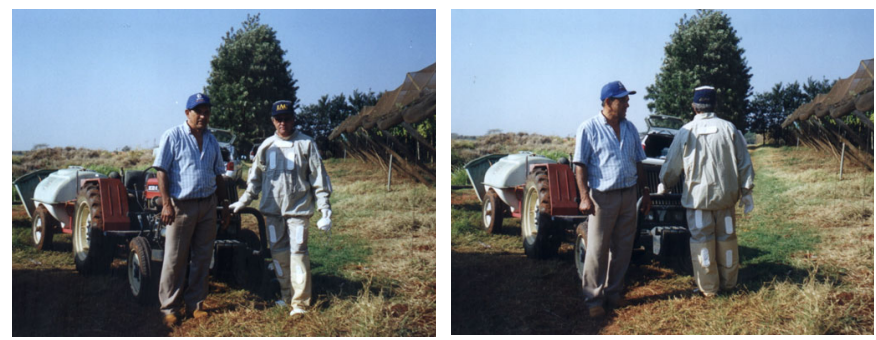

Figura 2. Detalhes do posicionamento dos amostradores durante a avaliação da exposição dérmica do aplicador na cultura da uva

Para avaliação da exposição na cabeça, os amostradores foram afixados sobre a parte superior de bonés ou da touca que compõe a roupa de segurança utilizada. $\mathrm{Na}$ face, foi afixado sobre uma máscara semi-facial descartável e nos pés (parte superior) diretamente na parte mediana das botas de borracha, sendo necessário, neste caso, um reforço na fixação, por meio de fita crepe nas pontas não absorventes do coletor. Nas mãos, foram utilizadas luvas de algodão como amostradores.

As aplicações na área experimental foram realizadas com calda contendo $6 \mathrm{~g} \mathrm{~L}^{-1}$ do fungicida Recop $(50 \% \mathrm{p} / \mathrm{p}$ de cobre metálico) com 3000 ppm do cátion $\mathrm{Cu}^{+2}$, utilizado como traçador. Para maior precisão na quantificação da calda recuperada, retirarou-se amostras de aproximadamente $70 \mathrm{~mL}$ nos tanques de cada tratamento. As avaliações foram realizadas nos dias 1 e 2 de setembro de 1999 e, durante o período de pulverização, as condições climáticas variaram conforme o descrito na Tabela 3.

Tabela 3. Condições climáticas durante o ensaio de exposição dérmica do aplicador

\begin{tabular}{|c|c|c|c|c|c|}
\hline \multirow{3}{*}{ Data } & \multicolumn{2}{|c|}{ Temperatura } & \multicolumn{2}{|c|}{ Umidade Relativa } & \multirow{3}{*}{$\begin{array}{c}\text { Velocidade } \\
\text { do Vento } \\
\left(\mathrm{km} \mathrm{h}^{-1}\right)\end{array}$} \\
\hline & Mínima & Máxima & Mínima & Máxima & \\
\hline & \multicolumn{2}{|c|}{${ }^{\circ} \mathrm{C}$} & \multicolumn{2}{|c|}{$\%$} & \\
\hline $1 / 9$ & 25,9 & 34,9 & 12 & 30 & 1 a 7 \\
\hline $2 / 9$ & 26,0 & 39,0 & 12 & 31 & 1 a 5 \\
\hline
\end{tabular}

O tempo de amostragem utilizado foi de 35 a 40 min de trabalho, período após o qual os coletores foram retirados dos trabalhadores, acondicionados em sacos plásticos, identificados e levados ao laboratório para quantificação do cátion $\mathrm{Cu}^{+2}$. Em algumas ocasiões durante o ensaio, foi necessário se remover os coletores da região das pernas e pés, antes do tempo de exposição determinado, pois os mesmos se molharam a ponto de saturação, ocasiões em que a pulverização foi interrompida, os coletores trocados e os tempos anotados. Todos os tratamentos foram repetidos 5 vezes.

No laboratório, realizou-se a recuperação do cátion nos coletores, através do método proposto por Machado Neto \& Matuo (1989). Aos sacos plásticos trazidos do campo, adicionaram-se 
$200 \mathrm{~mL}$ de uma solução de $\mathrm{HCl}$ 0,1 N agitando-a 20 vezes, mantendo-se o coletor imerso nessa solução, em repouso $24 \mathrm{~h}$. Após o período de repouso, os sacos plásticos foram novamente agitados 20 vezes e as soluções filtradas, através de papéis de filtro qualitativo, com diâmetro de $12 \mathrm{~cm}$, diretamente para dentro de copos coletores de $80 \mathrm{~mL}$. Finalmente, as soluções foram levadas à quantificação do $\mathrm{Cu}^{+2} \mathrm{em}$ espectrofotômetro de absorção atômica. Os resultados das leituras foram expressos em ppm de $\mathrm{Cu}^{+2}$.

As quantidades do cátion $\mathrm{Cu}^{+2}$ recuperadas nas áreas dos coletores $\left(70 \mathrm{~cm}^{2}\right)$ foram extrapoladas para as respectivas áreas das partes do corpo que representam (Tabela 2) para se obter sua exposição dérmica. No caso das mãos, não houve necessidade de extrapolação, pois a área das luvas foi considerada como a área das próprias mãos. Com esses valores e a concentração do traçador na calda original, estimou-se a exposição dérmica às caldas pulverizadas. Para facilitar a análise dos resultados, os dados foram agrupados de forma a representar 11 regiões do corpo e a exposição total foi obtida pela soma das exposições das 22 partes avaliadas (Tabela 2).

\section{RESULTADOS E DISCUSSÃO}

Os resultados das exposições dérmicas totais e nas diferentes partes do corpo, com as respectivas médias e desvios padrão, são apresentados na Tabela 4. Observa-se que os valores são bastante variáveis entre as repetições; entretanto, tal variação é esperada e se enquadra na faixa de limites aceitáveis estabelecidos nos Protocolos Padrões da Organização Mundial de Saúde (WHO, 1975 e WHO, 1982). Tais documentos estabelecem que, para um mesmo local do corpo, a variação não deve ser superior à média das repetições mais ou menos 3 vezes o desvio padrão.

Apesar da variação da exposição observada entre os tratamentos, constata-se que as áreas mais afetadas são as mãos, que receberam 46 a $69 \%$ da calda que atingiu o corpo do aplicador seguidas, normalmente, pela cabeça e dorso (17 a 44\%). A exposição significativamente maior na parte superior do corpo, era esperada pois, em função do sistema de plantio, a pulverização é realizada para cima, com gotas finas e, portanto, de deposição mais lenta, potencializando a exposição das áreas superiores do corpo. A grande exposição das mãos, em todos os tratamentos, pode ser explicada por uma possível contaminação durante o manuseio, reparos ou abastecimento dos equipamentos.

Pelos dados de exposição total, observa-se que o semiestacionário proporcionou exposições muito superiores aos demais tratamentos, ultrapassando a $3 \mathrm{~L}$ de calda por hora de trabalho, mesmo possuindo uma vazão do conjunto de bicos $\left(2,97 \mathrm{~L} \mathrm{~min}^{-1}\right)$ bem inferior à dos demais. Se se analisar os dados com base na porcentagem do volume pulverizado pelo conjunto de bicos que atinge o corpo do aplicador por minuto, observarse-ão valores de $0,019 \%$ para a barra adaptada, $0,020 \%$ para o turbo uva, $0,041 \%$ para o turbo adaptado e $1,79 \%$ para o semi-estacionário. Desta forma, a exposição proporcionada pelo semi-estacionário é 94,90 e 44 vezes maior que as proporcionadas, respectivamente, pela barra adaptada, turbo uva e turbo adaptado, o que pode ser explicado pela pulverização muito fina proporcionada pelos bicos Yamaho, que equipam este sistema de pulverização, aliada ao sentido de caminhamento do aplicador. Segundo Ramos et al. (2001) mais de 50\% do volume pulverizado por esses bicos, a pressões entre $703 \mathrm{e}$ $2813 \mathrm{kPa}$, são de gotas inferiores a $90 \mu \mathrm{m}$, o que lhes proporciona rápida perda de velocidade em função da pequena massa, baixa velocidade de queda e alto risco de deriva. Desta forma, grande parte das gotas pulverizadas não atinge o alvo, formando uma nuvem abaixo da videira, situação esta bastante visível e comum durante o ensaio. Em alguns países europeus e conforme Spraying Systems Co. (1999) é proibida a utilização de bicos de pulverização a pressões nas quais estes produzam mais que $10 \%$ do volume pulverizado com gotas inferiores a $115 \mu \mathrm{m}$, em função do seu potencial de deriva e contaminação. Assim, enquanto nos pulverizadores tratorizados os bicos de pulverização estão localizados a distâncias em torno de 2 a $3 \mathrm{~m}$ atrás do aplicador, no semi-estacionário essa distância é de $1 \mathrm{a} 2 \mathrm{~m}$ e os bicos são freqüentemente posicionados à sua frente, fazendo com que eles atravessem a nuvem de pulverização. Os efeitos desta técnica na contaminação do aplicador são visíveis nos dados da Tabela 2, uma vez que, enquanto nos pulverizadores tratorizados os principais pontos de exposição são localizados, no semi-estacionário eles se distribuem por praticamente todo o corpo.

Ainda analisando-se as porcentagens do volume pulverizado pelo conjunto de bicos que atingem o corpo do aplicador por minuto, observa-se que o turbo adaptado proporcionou exposições cerca de 2 vezes superiores à barra adaptada e ao

Tabela 4. Exposição dérmica potencial de aplicadores de agrotóxicos na cultura da uva

\begin{tabular}{|c|c|c|c|c|c|c|c|c|}
\hline \multirow{3}{*}{$\begin{array}{c}\text { Partes do } \\
\text { Corpo }\end{array}$} & \multicolumn{4}{|c|}{ Exposição do Aplicador $\left(\mathrm{mL} \mathrm{h}^{-1}\right)$} & \multirow{2}{*}{\multicolumn{2}{|c|}{ Barra Adaptada }} & \multirow{2}{*}{\multicolumn{2}{|c|}{ Semi-Estacionário }} \\
\hline & \multicolumn{2}{|c|}{ Turbo Adaptado } & \multicolumn{2}{|c|}{ Turbo Uva } & & & & \\
\hline & Média & Desvio-padrão & Média & Desvio-padrão & Média & Desvio-padrão & Média & Desvio-padrão \\
\hline Cabeça & 62,980 & 28,225 & 25,680 & 13,868 & 61,919 & 26,874 & 313,688 & 168,943 \\
\hline Rosto & 0,011 & 0,025 & 0,322 & 0,532 & 0,000 & 0,000 & 59,772 & 49,786 \\
\hline Pescoço & 0,002 & 0,004 & 0,000 & 0,000 & 0,000 & 0,000 & 3,487 & 6,007 \\
\hline Peito & 0,875 & 1,750 & 0,632 & 1,342 & 0,022 & 0,049 & 40,586 & 56,484 \\
\hline Dorso & 20,756 & 27,159 & 7,155 & 6,688 & 48,066 & 57,837 & 224,098 & 127,493 \\
\hline Ante-braço & 2,910 & 2,237 & 2,519 & 2,014 & 11,635 & 10,657 & 101,625 & 76,602 \\
\hline Braço & 2,274 & 2,479 & 2,369 & 2,400 & 7,045 & 13,516 & 373,342 & 327,207 \\
\hline Mão & 97,576 & 80,048 & 105,300 & 51,771 & 169,309 & 108,778 & 1475,547 & 847,478 \\
\hline Coxa & 1,183 & 2,366 & 4,287 & 5,614 & 3,745 & 5,175 & 213,430 & 275,320 \\
\hline Perna & 0,389 & 0,777 & 1,130 & 1,702 & 1,103 & 1,390 & 118,511 & 137,758 \\
\hline Pé & 2,524 & 3,280 & 2,249 & 1,319 & 1,091 & 1,424 & 285,449 & 221,442 \\
\hline Total & 191,478 & 98,414 & 151,643 & 43,238 & 303,935 & 188,686 & $3.209,535$ & $2.199,637$ \\
\hline
\end{tabular}


turbo uva, que tiveram porcentagens semelhantes. Tal fato pode ser explicado em função de um erro no projeto da turbina deste pulverizador, que fez com que a zona de admissão de ar estivesse muito próxima à barra de bicos, o que proporcionava áreas de turbulência e de ar descendente na região da nuvem de gotas, arrastando grande parte destas para fora do alvo. $\mathrm{Na}$ região de trabalho deste equipamento, a quantidade de gotas em suspensão foi superior à dos demais equipamentos tratorizados, aumentando a possibilidade do aplicador entrar em contato com as mesmas, arrastadas pelo vento, ao retornar à rua paralela.

\section{CONCLUSÕES}

Com base nos resultados obtidos, conclui-se que:

1. O uso de pulverizadores tratorizados proporcionou grande redução na exposição dérmica potencial do trabalhador, quando comparada com a obtida com o pulverizador semi-estacionário na cultura da videira, evidenciando maior segurança na utilização desses equipamentos.

2. Para todos os equipamentos tratorizados avaliados, as regiões mais expostas foram mãos, cabeça e dorso, devendo serem priorizadas no dimensionamento de equipamentos de proteção individual. Para o pulverizador semi-estacionário, a exposição foi elevada em praticamente todo o corpo.

\section{AGRADECIMENTOS}

Os autores agradecem à Fundação "Jorge Duprat Figueiredo" de Segurança e Saúde do Trabalho (FUNDACENTRO) e à Secretaria de Agricultura e Abastecimento do Estado de São Paulo (SAA-SP) que, através do Programa Segurança e Saúde do Trabalhador Rural (PSSTR), financiaram este projeto de pesquisa e, também, aos produtores rurais Sérgio Martins, Alcides Senerine, Shiro Kuroishi, Mauro Moura e Odemar Carvalho do Val, por sua valiosa atenção e colaboração no desenvolvimento das atividades de campo.

\section{LITERATURA CITADA}

Bonsall, J.L. Measurement of occupational exposure to pesticide. In: Turnbull, G.L. (ed.) Occupational hazards of pesticide use. London: Taylor \& Francis, 1985. p.13-33.

Durham, W.F., Wolfe, H.R. Measurement of the exposure of workers to pesticides. Bulletin of World Health Organization, Geneva, v.26, p.75-91, 1962.
IBGE - Instituto Brasileiro de Geografia e Estatísticas. Sistema IBGE de recuperação automática - SIDRA 97. Internet: http://www.sidra.ibge.gov.br, 6/99

Machado Neto, J.G. Estimativas do tempo de trabalho seguro e da necessidade de controle da exposição dos aplicadores de agrotóxicos. Jaboticabal: UNESP, 1997. 83p. Tese Livre-Docência

Machado Neto, J.G.; Matuo, T. Avaliação de um amostrador para estudo da exposição dérmica de aplicadores de defensivos agrícolas. Ciência Agronômica Jaboticabal, Jaboticabal, v.4, n.2, p.21-22, 1989.

Machado Neto, J.G.; Matuo, T.; Matuo, Y.K. Dermal exposure of pesticide applicators in staked tomato (Lycopersicon esculentum Mill) crops: Efficiency of a safety measure in the application equipment. Bulletin of Environmental Contamination and Toxicology, New York, v.48, n.4, p.529-534, 1992.

Machado Neto, J.G.; Matuo, T.; Matuo, Y.K. Efficiency of safety measures applied to a manual knapsack sprayer for paraquat application to maize (Zea mays L.) Archieves of Environmental Contamination and Toxicology, New York, v.35, n.4, p.698-701, 1998.

Maruyama, S. Fruticultura. 2.ed. Campinas: Instituto Campineiro de Ensino Agrícola. 1980. 385p.

Ramos, H.H.; Matuo, T; Bernardi, J.A. Características da pulverização produzida por bicos Yamaho da série ' $\mathrm{D}$ '. In: Congresso Brasileiro de Engenharia Agrícola, 30, 2001, Foz do Iguaçu. Anais... Foz do Iguaçu: SBEA. 2001. CD-Rom

Ramos, H.H.; Ramos, R.C.; Coelho, M.C.M.; Coelho, P.J. Avaliação do parque de pulverizadores em utilização no Estado de São Paulo. In: Congresso Brasileiro de Engenharia Agrícola, 27, 1998, Poços de Caldas. Anais... Poços de Caldas: SBEA. v.3, 1998. p.301-303.

Simão, S. Manual de fruticultura. São Paulo: Ceres. 1971. 530 p. Spraying Systems Co. Teejet - produtos de pulverização para agricultura, 46M-BR/P. Wheaton, Illinois. 1999. 104p.

Turnbull, G.L.; Sanderson, D.M.; Crome, S.J. Exposure to pesticide during application. In: Turnbull, G.L. (ed.) Occupational hazards of pesticide use. London: Taylor \& Francis. 1985. p.35-49.

WHO - World Health Organization. Survey of exposure to organophosphorus pesticide in agriculture standard protocol. Geneva: WHO, 1975. Document VBC/75.9

WHO - World Health Organization. Field surveys of exposure to pesticide standard protocol. Geneva: WHO, 1982. Document VBC/82.1 